\title{
Hyperbolic Multi-Monopoles With Arbitrary Mass
}

\author{
Lesley M. Sibner* and Robert J. Sibner ${ }^{\dagger \dagger}$
}

October 1, 2012

\begin{abstract}
On a complete manifold, such as $\mathbb{R}^{3}$ or hyperbolic space $\mathbb{H}^{3}$, the limit at infinity of the norm of the Higgs field $\Phi$ is called the mass of the monopole. We show the existence, on $\mathbb{H}^{3}$, of monopoles with given magnetic charge and arbitrary mass. Previously, aside from charge one monopoles, existence was known only for monopoles with integral mass (since these arise from $\mathrm{U}(1)$ invariant instantons on $\mathbb{R}^{4}$ ). The method of proof is based on Taubes' gluing procedure, using well-separated, explicit, charge one monopoles. The analysis is carried out in a weighted Sobolev space and necessitates eliminating the possibility of point spectra.
\end{abstract}

${ }^{*}$ Department of Mathematics, Polytechnic Institute of NYU, Brooklyn, NY 11201, USA lsibner@duke.poly.edu

${ }^{\dagger}$ Department of Mathematics, City University of New York, Graduate Center and Brooklyn College, Brooklyn, NY 11210, USA. rsibner@gc.cuny.edu

${ }^{\ddagger}$ Research partially supported by PSC-CUNY grant 69570-00-38 


\section{Higgs Field and Holonomy}

The characteristic numbers of a magnetic monopole on a 3-manifold $\mathrm{M}$ (without boundary) are its integer valued magnetic charge $\mathrm{k}$ and the asymptotic limit $\mathrm{m}$ of the length of its Higgs field at an end. (This limit is referred to as the mass of the Higgs field.) For $M=\mathbb{R}^{3}$, using scaling techniques, one can assume without loss of generality that $m=1$, but on hyperbolic space $\mathbb{H}^{3}$ scaling is not possible. Aside from known explicit charge one monopoles, most attention has been given to monopoles on $\mathbb{H}^{3}$ with integral $\mathrm{m}$. The reason for this is that one can (see Atiyah $[\mathrm{A}]$ ), up to conformal equivalence, consider $\mathbb{H}^{3}$ as $\mathbb{R}^{4} \backslash \mathbb{R}^{2}$ modulo a $\mathrm{U}(1)$ action leaving $\mathbb{R}^{2}$ invariant. Then any $\mathrm{U}(1)$ invariant instanton on $\mathbb{R}^{4}$ with fixed set $\mathbb{R}^{2}$ produces a hyperbolic monopole on $\mathbb{H}^{3}$ with integral $\mathrm{m}$. Conversely, a hyperbolic monopole on $\mathbb{H}^{3}$ can be lifted to an instanton on $\mathbb{H}^{3} \times \mathrm{S}^{1}$, resulting in a hyperbolic caloron (see $[\mathrm{A}, \mathrm{L}, \mathrm{H}$, $\mathrm{MS}]$ ). We have shown previously that it is precisely the hyperbolic monopoles with integral mass $m$ which allow extensions to instantons on all $\mathbb{R}^{4}$. To understand this better, we recall [SS1, SS2] that any $\mathrm{L}^{2}$ (singular) connection on $\mathbb{R}^{4} \backslash \mathbb{R}^{2}$ (not necessarily satisfying any field equations) has a well-defined limit holonomy which is constant along the singular set $\mathbb{R}^{2}$. (The $\mathrm{L}^{2}$ connections are classified by the space of flat connections.) The limit holonomy corresponds (via Atiyah's construction) to the mass of the corresponding configuration on $\mathbb{H}^{3}$, with integral mass corresponding to integral holonomy. The integrality of the holonomy is a necessary and sufficient condition for the connection on $\mathbb{R}^{4} \backslash \mathbb{R}^{2}$ to extend across the $\mathbb{R}^{2}$. On the other hand, however, from the point of view of the 3 -manifold $\mathbb{H}^{3}$, the restriction to integral $\mathrm{m}$ seems clearly artificial. Indeed, on $\mathbb{H}^{3}$ it makes analytic sense to prescribe the mass as any positive real number. This view was already pointed out by Atiyah in his 1984 publication [A], and is supported by the example [FHP1, FHP2] of a U(1) invariant instanton on $\mathbb{R}^{4} \backslash \mathbb{R}^{2}$ with holonomy $1 / 2$ which does not extend.

We recall some basic definitions. A connection on an $\mathrm{SU}(2)$ vector bundle over a simply connected 3-manifold $\mathrm{M}$ can be pulled back to an su(2) valued (connection) 1-form $\mathrm{A}$ on $\mathrm{M}$ and gives rise to a covariant derivative $d_{A}=d+[A$,$] . A Higgs monopole on \mathrm{M}$ (see [JT]) is a configuration pair $c=(\Phi, A)$ where $\Phi$ is an $\operatorname{su}(2)$ valued function on $\mathrm{M}$. (If $\mathrm{M}$ is not simply connected, these pullbacks are local.) The curvature of the connection is given by $F=d A+\frac{1}{2}[A, A]$ and the pair satisfies the Bogomolny monopole equations [Bo]:

$$
d_{A} \Phi=* F_{A}
$$

The solutions of (1.1) are the absolute minima of the Yang-Mills-Higgs action functional

$$
\mathcal{Y} \mathcal{M H}(c)=\frac{1}{2} \int_{M}\left(\left|F_{A}\right|^{2}+\left|d_{A} \Phi\right|^{2}\right) d V
$$

This theory becomes interesting if $M$ has an "end" (such as $\mathbb{R}^{3}$ or $\mathbb{H}^{3}$ ) in which case the natural boundary and topological conditions are given by prescribing the mass of the monopole

$$
m=\lim _{|x| \rightarrow \infty}|\Phi(x)|
$$


and the magnetic charge

$$
k=\frac{1}{4 \pi m} \int_{M} \operatorname{tr}\left(F_{A} \wedge d_{A} \Phi\right)
$$

Our main result is the following existence theorem for multi-monopoles on $\mathbb{H}^{3}$ of arbitrary mass.

Theorem. There exists a smooth configuration $c=(\Phi, A)$ on $\mathbb{H}^{3}$, having prescribed magnetic charge $k \in \mathbb{Z}$, prescribed mass $m>0$ at infinity, and satisfying the Bogomolny monopole equation (1.1).

From the preceding remarks, noting the identification of mass for monopoles on $\mathbb{H}^{3}$ with holonomy for instantons on $\mathbb{H}^{3} \times S^{1}$, it is clear that one has immediately the following:

Corollary. There exists a hyperbolic caloron (on $\mathrm{M}=\mathbb{H}^{3} \times S^{1}$ ) having prescribed topological charge (instanton number) $\mathrm{N}$ and prescribed holonomy (not necessarily integral).

In the mid 1970's, by making a symmetry ansatz, an explicit SU(2) Bogomolny monopole solution on $\mathbb{R}^{3}$ was obtained by Prasad and Sommerfeld [PS]. (Earlier, Dirac [Di] had obtained a $\mathrm{U}(1)$ monopole on $\mathbb{R}^{3}$.) Later, Chakrabarti $[\mathrm{C}]$ wrote down an explicit solution on hyperbolic 3-space $\mathbb{H}^{3}$ (see also Braam [Br]). All of these explicit solutions were of charge $k=1$. The explicit solutions on $\mathbb{R}^{3}$ became "building blocks" in the next development. With a procedure that has, by now, become standard, Taubes [JT] constructed monopoles on $\mathbb{R}^{3}$ (with arbitrary charge) by a "patching argument." The basic idea (which we will follow in the sequel) is to construct an "approximate" monopole and then perturb it to a true monopole using some version of the Implicit Function Theorem. This method gives solutions which are (for given charge) absolute minima of the action functional. (Taubes went on to utilize this program to show that the moduli space of self dual instantons on appropriate 4-manifolds was non empty.) The program has been used by Floer [F1, F2], Ernst [E] and Durenard $[\mathrm{Du}]$ in the construction of monopoles on 3-manifolds with Euclidean ends. These last results, together with our Theorem, naturally suggest (with some restrictions) the following:

Conjecture. Let $M$ be a Riemannian 3-manifold with $N$ hyperbolic ends. There exists a smooth configuration on $M$, satisfying the Bogomolny equations, having prescribed magnetic charge $k \in \mathbb{Z}$ and prescribed asymptotic limits $m_{j}, j=1, \ldots, N$ at the ends.

To prove the theorem, we follow a program similar to that developed by Taubes and discussed above. We work directly on the space $\mathbb{H}^{3}$ and do not require any assumption on the magnitude of $m$ nor on the charge $k$.

In Section 2 we construct an approximate solution and define the weighted Sobolev space in which it lies. In Section 3 the perturbation problem is derived. Sections 4 and 5 are 
devoted to showing that the origin is not contained in the spectrum of the various operators. In section 4 we use results of Mazzeo $[\mathrm{M}]$ to show the absence of eigenvalues. In section 5 we show that the essential spectra have positive lower bounds. Then, in Section 6, a lower bound estimate is obtained for a linearized operator. A continuity argument completes the proof of existence.

In light of recent interest in the classification of hyperbolic monopoles by the asymptotic values of their Higgs fields, it is perhaps worth noting that all the monopoles that we construct are of Dirac type at infinity.

We remark that this general method is, by now, standard and has been exploited successfully in many situations in which the lower bound can be established. However, in our case, the a priori bound is false in the usual $\mathrm{L}^{2}$ Sobolev spaces over $\mathbb{H}^{3}$ and, in order to work directly on $\mathbb{H}^{3}$, one must use weighted Sobolev spaces.

The authors wish to acknowledge and thank the graciousness of the Max Planck Institute in Bonn, Germany and the Institut des Hautes Etudes Scientifique in Bures-sur-Yvette, France for their hospitality during the periods when preliminary work on this project was carried out.

\section{The Approximate Monopole}

The basic $\mathrm{SU}(2)$, charge $k=1$ and mass $\mathrm{m}$, monopole on $\mathbb{H}_{3}$ can be written down explicitly [C]. To obtain an approximate monopole of given charge, $k$, first choose $k$ points $x_{1}, \ldots, x_{k}$ as centers of charge one monopoles, requiring that the distance between centers be greater than $6 R$ where $R \geq 1$ is a constant to be chosen later. The distance between the centers $x_{i}$ are also chosen so that, denoting by $B_{\rho}^{\mathrm{i}}$ the ball of radius $\rho$ about $x_{i}$, we can choose geodesic spherical coordinates $\left(r_{i}, \theta_{i}, \chi_{i}\right)$ centered at $x_{i}$ so that the half rays $\theta_{i}=0$ and $\theta_{i}=\pi$ do not intersect the closure of any of the sets $U_{R}^{j}=B_{2 R}^{j} \backslash B_{R}^{j}$ for $j \neq i$.

Let $c_{i}=\left(\Phi_{i}, A_{i}\right)$ be the basic Chakrabarti, charge one, monopole at $x_{i} \in \mathbb{H}^{3}[\mathrm{C}]$.

$$
\left\{\begin{array}{l}
\Phi_{i}=\left(\alpha \operatorname{coth} \alpha r_{i}-\operatorname{coth} r_{i}\right) \hat{i} \\
A_{i}=\frac{\alpha \sinh r_{i}}{\sinh \alpha r_{i}}\left(d \theta_{i} \hat{j}+\sin \theta_{i} d \chi_{i} \hat{k}\right)+\left(1-\cos \theta_{i}\right) d \chi_{i} \hat{i}
\end{array}\right.
$$

where we have written $\alpha=m+1$ and the Pauli matrices $\hat{i}, \hat{j}, \hat{k}$ are a basis for $\mathrm{su}(2)$.

In the neighborhood of infinity $N_{\infty}=\mathbb{H}^{3} \backslash \cup_{i=1}^{k} B_{R}^{i}$, we take a U(1)-Dirac monopole $c_{\infty}=\left(\Phi_{\infty}, A_{\infty}\right)$ where 


$$
\begin{aligned}
& \Phi_{\infty}=\left\{(\alpha-1)+\left(1-\operatorname{coth} r_{1}\right)+\ldots+\left(1-\operatorname{coth} r_{k}\right)\right\} \hat{i} \\
& \left.A_{\infty}=\left\{\left(1-\cos \theta_{1}\right) d \chi_{1}+\ldots+\left(1-\cos \theta_{k}\right) d \chi_{k}\right)\right\} \hat{i} .
\end{aligned}
$$

In any system of geodesic polar coordinates $(r, \theta, \chi)$ the metric is given by

$$
\begin{aligned}
d s^{2} & =d r^{2}+\sinh ^{2} r d \Omega^{2} \\
& =d r^{2}+\sinh ^{2} r d \theta^{2}+\sinh ^{2} r \sin ^{2} \theta d \chi^{2}
\end{aligned}
$$

so that the volume element is given by $d V=\sinh ^{2} r \sin \theta d r d \theta d \chi$. In the metric induced on the cotangent space

$$
|d r|=1,|d \theta|=(\sinh r)^{-1} \text { and }|d \chi|=(\sinh r \sin \theta)^{-1}
$$

While the Higgs action of $c_{i}(1 \leq u \leq k)$ is finite, the action of $c_{\infty}$ on $\mathbb{H}^{3}$ is not finite because of the singular behaviour of $F_{A_{\infty}}$ at the points $x_{i}$. However the restriction of $F_{A_{\infty}}$ to $N_{\infty}$ does have finite action. In the gauge of $(2.1 \mathrm{a}) c_{i}$ has a Dirac string singularity along the half ray $\theta_{i}=\pi$. (note that $\left|1-\cos \theta_{i}\right|\left|d \chi_{i}\right| \rightarrow \infty$ as $\theta_{i} \rightarrow \pi$ ). However, since the holonomy around the string is integral, the codimension two removable singularity theorem [SS1,SS2] ensures that $c_{i}$ is gauge equivalent to a smooth configuration. The same is true of $c_{\infty}$ in $N_{\infty}$.

We emphasize that $c_{\infty}$ and each of the $c_{i}$ are solutions of the Bogomolny equations:

$$
d_{A} \Phi=* F_{A} .
$$

In particular, this is true for $c_{i}$ on $B_{2 R}^{i}$ and $c_{\infty}$ on $N_{\infty}$, so that if we glue them together by a partition of unity $\left\{\lambda_{1}, \ldots, \lambda_{k}, \lambda_{\infty}\right\}$ subordinate to the covering of $\mathbb{H}^{3}$ by $N_{\infty}$ and the $B_{2 R}^{i}, 1 \leq i \leq k$, we obtain an "approximate monopole" $c_{0}=\left(\Phi_{0}, A_{0}\right)$ :

$$
\left\{\begin{array}{l}
\Phi_{0}=\lambda_{\infty} \Phi_{\infty}+\sum_{i=1}^{k} \lambda_{i} \Phi_{i} \\
A_{0}=\lambda_{\infty} A_{\infty}+\sum_{i=1}^{k} \lambda_{i} A_{i}
\end{array}\right.
$$

By its construction, $c_{0}$ satisfies the monopole equation (2.3) in each $B_{R}^{i}$ and in $N_{\infty} \backslash$ $\cup_{i=1}^{k} B_{2 R}^{i}$. We need to estimate the deviation of $c_{0}$ from a solution in the intersections $U_{R}^{i}=$ $B_{2 R}^{i} \backslash B_{R}^{i}$. Note that, in the partition of unity construction, at most two $\lambda$ 's can be non-zero simultaneously. In particular, in $U_{R}^{i}$ we have $\lambda_{i}+\lambda_{\infty}=1$. Moreover, for $x \in U_{R}^{i}$ and $k \neq i$, one has $r_{k}(x)>4 R$. In $U_{R}^{i}$

$$
\begin{gathered}
d_{A_{0}} \Phi_{0}-* F_{A_{0}}=\lambda_{\infty}\left(1-\lambda_{\infty}\right)\left\{\left[A_{\infty}-A_{i}, \Phi_{\infty}-\Phi_{i}\right]+*\left[A_{\infty}-A_{i}, A_{\infty}-A_{i}\right]\right\} \\
+d \lambda_{\infty}\left(\Phi_{\infty}-\Phi_{i}\right)-*\left(d \lambda_{\infty} \wedge\left(A_{\infty}-A_{i}\right)\right)
\end{gathered}
$$

The terms in (2.5) can be estimated, using (2.2), to obtain the pointwise bound in $U_{R}^{i}$ : 


$$
\left|d_{A_{0}} \Phi_{0}-* F_{A_{0}}\right| \leq K\left(e^{-\alpha r_{i}}+e^{-4 R}\right) \text { with } K \text { independent of } R \geq 1 .
$$

This can be done in the subdomain of $U_{R}^{i}$ where $0 \leq\left|\theta_{i}\right|<3 \pi / 4$ in the gauge in which the configuration is represented by (2.1ab). In the overlapping region where $\pi / 4<\left|\theta_{i}\right| \leq \pi$ one should choose a gauge in which the string is given by $\theta_{i}=0$. See $[\mathrm{JT}]$ for a discussion of inverting strings and also for the fact that the existence of local smoothing gauges implies the existence of a global smoothing gauge. (The estimate (2.6) is gauge invariant.) In computing bounds for each term of (2.5) in the region $U_{R}^{i}$, one finds that it is the term $\left|A_{\infty}-A_{i}\right|$ that decays most slowly and gives the upper bound (2.6).

We define the weighted spaces $L_{\beta}^{p}$ on $q$-forms, as the completion of $C_{0}^{\infty}\left(\wedge^{q}\right)$ in the norm:

$$
\|\omega\|_{p, \beta}=\left(\int_{\mathbb{H}^{3}}|\omega|^{p} \cosh ^{2} \beta r d V\right)^{1 / p}
$$

We assume $\beta<1$ which ensures that our appproximate monopole $c_{0}$ has finite weighted action; namely,

$$
\mathcal{Y} \mathcal{M} \mathcal{H}_{\beta}\left(c_{0}\right)=\frac{1}{2} \int_{\mathbb{H}^{3}}\left(\left|F_{A_{0}}\right|^{2}+\left|d_{A_{0}} \Phi_{0}\right|^{2}\right) \cosh ^{2} \beta r d V<\infty .
$$

We can now easily show

Proposition 2.7. If $\beta<\min (1, m)$, there is a constant $c>0$ depending on $m$ but not on $R$, such that

$$
\left\|d_{A_{0}} \Phi_{0}-* F_{A_{0}}\right\|_{2, \beta} \leq K e^{-c R}
$$

Proof: Outside $\cup_{i=1}^{k} U_{R}^{i}, d_{A_{0}} \Phi_{0}-* F_{A_{0}}$ vanishes. Using (2.6) in $U_{R}^{i}$ and recalling that $\alpha=$ $m+1$, we see that

$$
\begin{aligned}
\left\|d_{A_{0}} \Phi_{0}-* F_{A_{0}}\right\|_{2, \beta}^{2} & \leq K\left\{\int_{R}^{2 R} e^{(-2 m+2 \beta) r_{i}} d r_{i}+e^{-8 R}\left(\int_{R}^{2 R} e^{2 \beta+2) r_{i}} d r_{i}\right)\right\} \\
& \leq K\left\{e^{4(\beta-m) R}+e^{4(\beta-1) R}\right\}
\end{aligned}
$$

The constant $c$ will have the right sign if $\beta<m$ and $\beta<1$. This proves the proposition.

\section{The Perturbation Problem}

We now look for solutions of (1.1) of the form $c=c_{0}+\zeta=\left(\Phi_{0}+\varphi, A_{0}+a\right)$ with $c_{0}$ the approximate monopole of $\S 2$. Following Floer [F2], we expand (1.1) in a Taylor expansion to obtain

$$
L c=d_{A} \Phi-* F_{A}=L c_{0}+D_{L} \zeta+\sigma(\zeta, \zeta)
$$


Here, $D_{L}$ is the linearization of $L$ at $c_{0}$; i.e.,

$$
D_{L} \zeta=D_{L}(\varphi, a)=-* d_{A_{0}} a+d_{A_{0}} \varphi-\left[\Phi_{0}, a\right]
$$

The quadratic term $\sigma(\zeta, \zeta)$ is defined by a bilinear bundle map of $\wedge^{0} \oplus \wedge^{1}$ into $\wedge^{1}$.

We work in the weighted Sobolev spaces described in $\S 2$. Throughout, the mass is a fixed arbitrary positive number and the constant $\beta$ appearing in the weight factor satisfies $0<\beta<\min (1, m)$. In the weighted Sobolev space, the adjoint of the operator $d_{A_{0}}$ on forms $\omega \in \wedge^{q}$ is:

$$
d_{A_{0}}^{\dagger} \omega=\left(\cosh ^{-2} \beta r\right) d_{A_{0}}^{*}\left(\left(\cosh ^{2} \beta r\right) \omega\right)
$$

where $d_{A_{0}}^{*}$ is the ordinary $L^{2}$ adjoint. To obtain ellipticity, we add a "slice" condition:

$$
D_{S} \zeta=D_{S}(\varphi, a)=d_{A_{0}}^{\dagger} a-\left[\Phi_{0}, \varphi\right]=0
$$

This together with (3.2) gives an elliptic operator on pairs

$$
\delta=\left(D_{S}, D_{L}\right): \wedge^{0} \oplus \wedge^{1} \rightarrow \wedge^{0} \oplus \wedge^{1}
$$

defined by

$$
\delta \zeta=\left(\delta_{A_{0}}-a d \Phi_{0}\right)(\varphi, a)
$$

where we have written $\delta_{A_{0}}(\varphi, a)=\left(d_{A_{0}}^{\dagger} a, d_{A_{0}} \varphi-* d_{A_{0}} a\right)$ and $a d \Phi_{0}(\varphi, a)=\left(\left[\Phi_{0}, \varphi\right],\left[\Phi_{0}, a\right]\right)$.

To solve the Bogomolny equations (1.1), we want to find solutions $\zeta=(\varphi, a)$ of

$$
\delta \zeta+\zeta \# \zeta=G_{0}
$$

where $\zeta \# \zeta=(0, \sigma(\zeta, \zeta))$ and $G_{0}=\left(0,-L c_{0}\right)$ is sufficiently small in appropriate norms. As in [T, FU], we look for a solution perpendicular to the kernel of $\delta$ by setting $\zeta=\delta^{\dagger} \eta$ and solving for $\eta=(\psi, b), \psi \in \wedge^{0}$ and $b \in \wedge^{1}$,

$$
\delta \delta^{\dagger} \eta+\delta^{\dagger} \eta \# \delta^{\dagger} \eta=G_{0} .
$$

Since $a d \Phi_{0}$ is skew adjoint,

$$
\delta^{\dagger}=\delta_{A_{0}}^{\dagger}+a d \Phi_{0}
$$

so that

$$
\delta \delta^{\dagger}=\left(\delta_{A_{0}}-a d \Phi_{0}\right)\left(\delta_{A_{0}}^{\dagger}+a d \Phi_{0}\right)=\delta_{A_{0}} \delta_{A_{0}}^{\dagger}-\left(a d \Phi_{0}\right)^{2}+E
$$

where $E \eta=\left(-*\left[d_{A_{0}} \Phi_{0}, * b\right],\left[d_{A_{0}} \Phi_{0}, \psi\right]-*\left[d_{A_{0}} \Phi_{0}, b\right]\right)$. 
A computation shows that the $L_{\beta}^{2}$ adjoint of $\delta_{A_{0}}$ is:

$$
\delta_{A_{0}}^{\dagger} \eta=\delta_{A_{0}}^{\dagger}(\psi, b)=\left(d_{A_{0}}^{\dagger} b, d_{A_{0}} \psi-*\left(\cosh ^{-2} \beta r\right) b\right) d_{A_{0}}\left(\cosh ^{2} \beta r\right) b
$$

It follows that

$$
\delta_{A_{0}} \delta_{A_{0}}^{\dagger}(\eta)=d_{A_{0}} d_{A_{0}}^{\dagger}+d_{A_{0}}^{\dagger} d_{A_{0}}+T_{A_{0}} \eta=\Delta_{A_{0}} \eta+T_{A_{0}} \eta
$$

where, $\Delta_{A_{0}} \eta=\Delta_{A_{0}}(\psi, \mathrm{b})=\left(\Delta_{A_{0}} \psi, \Delta_{A_{0}} \mathrm{~b}\right)$ and

$$
T_{A_{0}} \eta=\left(*\left[F_{A_{0}}, b\right], *\left[F_{A_{0}}, \psi\right]-2 \beta \tanh \beta r\left\{*\left(d r \wedge * d_{A_{0}} b\right)+d_{A_{0}}^{*}(b \wedge d r)\right\}\right) .
$$

When $A_{0}=0$, these operators reduce, respectively, to the (weighted) scalar Laplacian $\Delta$ and to

$$
T=\left(0, T_{1}\right) \text { where } T_{1}=-2 \beta \tanh \beta r\left\{* d r \wedge * d b+d^{*}(d r \wedge b)\right\}
$$

Writing

$$
\Delta_{A_{0}}=\Delta+S \text { and } T_{A_{0}}=T+R
$$

one finds

$$
S \eta=\left(S_{0} \psi, S_{1} b\right) \text { where } S_{0} \psi=d^{\dagger}\left[A_{0}, \psi\right]+\left[A_{0}, d^{\dagger} b+\left[A_{0}, b\right]\right] \text { and }
$$

$S_{1} b=d^{\dagger}\left[A_{0}, b\right]+d\left(*\left[A_{0}, * b\right]\right)+*\left[A_{0}, *\left(d b+\left[A_{0}, b\right]\right)\right]+\left[A_{0}, d^{\dagger} b+*\left[A_{0}, * b\right]\right]$

$$
R \eta=\left(*\left[F_{A_{0}}, b\right], *\left[F_{A_{0}}, \psi\right]+* 2 \beta \tanh \beta r\left\{\left[A_{0}, *(d r \wedge b)\right]-d r \wedge *\left[A_{0}, b\right]\right\}\right)
$$

Also, for $A_{0}=0$ we see from (3.10) that

$$
\delta_{0} \delta_{0}^{\dagger}=\Delta+T
$$

Moreover, using (3.10), (3.13) and (3.16),

$$
\delta_{A_{0}} \delta_{A_{0}}^{\dagger}=\Delta_{A_{0}}+T_{A_{0}}=\Delta+R+S+T=\delta_{0} \delta_{0}^{\dagger}+R+S
$$

Recall from (2.1b) that, near infinity, $c_{0}=\left(\Phi_{0}, A_{0}\right)=\left(\Phi_{\infty}, A_{\infty}\right)$ so that this configuration decays exponentially in the sense that

$$
\left|d \Phi_{0}\right|,\left|A_{0}\right|,\left|F_{A_{0}}\right| \leq e^{-c|x|} \text { for large }|x|
$$

As a result, an examination of the various "remainder" operators above shows that they are all rapidly decaying.

Proposition 3.18. $|(R+S+T+E) \eta| \leq \tau(r)(|\eta|+|\nabla \eta|)$ with $\tau(r)$ decaying exponentially at infinity. 


\section{The Absence of Point Spectra}

We will see in Section 5 that the weight factor, $\cosh ^{2} \beta r$, shifts the essential spectrum of the Laplacian to the right. The same is true for the operator $\delta \delta^{\dagger}$ which differs from the Laplacian by a first order partial differential operator. To resolve the question of the possible emergence of eigenvalues when the weight is introduced, we note that the weighted norm comes from the spherically symmetric metric

$$
d s^{2}=d r^{2}+f^{2}(r) d \Omega^{2}
$$

where $f(r)=\cosh \beta r \sinh r$. The non-zero components of the Riemann curvature tensor are:

$$
\frac{-f^{\prime \prime}}{f} \text { and } \frac{1-f^{\prime 2}}{f^{2}} \text {. }
$$

Since $f(0)=f^{\prime \prime}(0)=0$ and $f^{\prime}(0)=1$, the metric is non-singular, and the sectional curvatures are bounded. Therefore, in general (with $d_{B}^{*}$ the adjoint of $d_{B}$ ).

(i) $\quad d_{B_{i}}-\nabla_{i}=B_{i}$

(ii) $\quad \Delta_{B}=d_{B} d_{B}^{*}+d_{B}^{*} d_{B}=\nabla^{*} \nabla+Q$

where $Q$ involves $F_{B}$ and Ricci curvature and satisfies: $|Q u| \leq c(|u|+|\nabla u|)$. (See [JT] for more discussion.)

Let $M=\left(\mathbb{H}^{3}, g\right)$ where $g$ is the metric above. Let $E$ be a vector bundle over $M$ with structure group $\mathrm{SU}(2)$. In the following sections, E will be either the bundle of zero-forms or of one-forms.

Theorem 4.1. (Mazzeo $[\mathrm{M}])$ Let $u \in C^{\infty}(M, E)$. Suppose $u=0$ when $r \leq r_{0},|u|=O\left(e^{-c r}\right)$ for some $c>0$, and $u$ is in the $L^{2}$ domain of $\nabla^{*} \nabla$. Then, for $r_{0}$ and $t$ sufficiently large and $C$ independent of $t$,

$$
t^{3} \int e^{2 t r}|u|^{2} d V+t \int e^{2 t r}|\nabla u|^{2} d V \leq C \int e^{2 t r}\left|\nabla^{*} \nabla u\right|^{2} d V
$$

Corollary 4.2. Let $u$ be as in Theorem 4.1. If, in addition

$$
\left|\nabla^{*} \nabla u\right| \leq k(|u|+|\nabla u|)
$$

then $u \equiv 0$.

Corollary 4.3. Let $w \in L_{\beta}^{2}\left(\mathbb{H}^{3}, E\right)$ be an eigenfunction satisfying

$$
L w=\lambda w
$$

where $L=\nabla^{*} \nabla+Q$ where $Q$ satisfies the bound in $(i i)$ above. Then $w \equiv 0$.

By Proposition (3.18) we know that all of the operators we will consider in the following sections (namely $\Delta, \delta_{0} \delta_{0}^{\dagger}, \delta_{A_{0}} \delta_{A_{0}}^{\dagger}$, or $\delta \delta^{\dagger}$ ) satisfy the hypotheses of Corollary 4.3 and, 
consequently, have no point spectrum. In all cases, the spectrum consists only of essential spectrum. Nevertheless, for consistency with quoted results and for clarity of exposition, although they coincide in our case, in the following we will use $\sigma$ to denote the spectrum of an operator and $\sigma_{\infty}$ its essential spectrum.

\section{The Essential Spectrum}

To calculate the essential spectrum, we apply Donnelly's method [Do] of separation of variables to the weighted space and to sections (zero forms or one forms) with $\mathrm{su}(2)$ valued coefficients.

Recall that $\Delta_{p}=d^{\dagger} d+d d^{\dagger}(p=0$ or 1$)$ is the weighted self-adjoint Laplacian on $L_{\beta}^{2}\left(\mathbb{H}^{3}\right)$ with domain $L_{\beta}^{2,2}\left(\mathbb{H}^{3}\right)$ and $\delta_{0} \delta_{0}^{\dagger}$ is the Floer operator of $(3.6)$ (with $A_{0}=0$ ).

\section{Proposition 5.1.}

(a) $\sigma\left(\Delta_{0}\right)=\left[(1+\beta)^{2}, \infty\right)$

(b) $\sigma\left(\Delta_{1}\right)=\left[\beta^{2}, \infty\right)$

(c) $\sigma\left(\delta_{0} \delta_{0}^{\dagger}\right)=\left[\beta^{2}, \infty\right)$

We emphasize that this result applies to the operators evaluated at the zero connection.

We make extensive use of the following proposition which tells us that, as long as the coefficients tend to zero at infinity, a smooth first order operator $C$ is relatively compact with respect to a self-adjoint second order elliptic operator $L$; its addition to $L$ does not change the essential spectrum. We state this in the form which will be applied.

Proposition 5.2. Let $L$ be an elliptic, second order, self-adjoint operator on $L_{\beta}^{2}(M)$, where $M$ is either the non-negative reals $\mathbb{R}^{+}$, or $\mathbb{H}^{3}$. Assume that the domain of $L$ is $\mathcal{H}_{B}=L_{\beta, B}^{2,2}$ (using covariant derivatives at B). Let $C=\sum a_{i} \frac{\partial}{\partial x_{i}}+b$ where the coefficients are smooth functions and $\tau(r)=\max _{|x| \geq r}\left(\left|a_{i}\right|,|b|\right)$. If $\tau(r)$ tends to zero as $r$ tends to infinity then $\sigma_{\text {ess }}(L)=\sigma_{\text {ess }}(L+C)$.

Proof. For some (and hence every) $z$ in the resolvent of $L, R=(L-z I)^{-1}$ is a bounded operator from $L_{\beta}^{2}$ to $\mathcal{H}_{B}$ and hence, for some constant $k$,

$$
\|R f\|_{\mathcal{H}_{B}} \leq k\|f\|_{L_{\beta}^{2}}
$$

Choose an exhaustion $\left\{M_{n}\right\}$ of $M$ and cutoff functions $u_{n}$ with supp $u_{n} \subset M_{n}, u_{n} \equiv 1$ on $M_{n-1}$ and $\left|\nabla u_{n}\right| \rightarrow 0$ as $n \rightarrow \infty$. By Rellich's lemma, $D_{n}=u_{n} C R$ is compact on $L_{\beta}^{2}(M)$.

Claim. $C$ is relatively compact with respect to $L$; i.e., $D=C R$ is compact on $L_{\beta}^{2}(M)$. This 
follows from the inequality:

$$
\begin{gathered}
\left\|\left(D-D_{n}\right) f\right\|_{L_{\beta}^{2}(M)}^{2}=\left\|\left(1-u_{n}\right) C R f\right\|_{L_{\beta}^{2}(M)}^{2} \\
\leq \int_{M \backslash M_{n-1}}|C R f|^{2} \cosh ^{2} \beta r d V \\
\leq k^{\prime} \tau\left(r_{n}\right)\|R f\|_{L_{\beta}^{1,2}(M)}^{2} \\
\leq k^{\prime \prime} \tau\left(r_{n}\right)\|f\|_{L_{\beta}^{2}(M)}^{2}
\end{gathered}
$$

which shows that $D_{n}$ converges to $D$ in norm. The result now follows from

Theorem. (Weyl, cf. [RS] Corollary 2, IV p. 113) If $L$ is as in Proposition 5.2 and $\exists z \in \mathbb{C}$ such that $C(L-z I)^{-1}$ is compact, then $\sigma_{\text {ess }}(L)=\sigma_{\text {ess }}(L+C)$.

The prototype of ordinary differential operators which arise in the Donnelly decomposition is

$$
\mathcal{D} f=-\frac{d^{2} f}{d x^{2}}-2 \gamma \frac{d f}{d x}+c(x) f
$$

where $c(x)$ is rapidly decaying, $\gamma>0$ is constant, and $f \in L^{2}\left(R^{+}, e^{2 \gamma x} d x\right)$.

Lemma 5.4 Up to compact perturbation, $\mathcal{D}$ as defined in (5.3) is unitarily equivalent to the multiplication operator, $(\mathcal{M} f)(x)=\left(x^{2}+\gamma^{2}\right) f(x)$ acting on $L^{2}\left(R^{+}, d x\right)$. Therefore, $\sigma_{\text {ess }}(\mathcal{D})=\sigma(\mathcal{D})=\left[\gamma^{2}, \infty\right)$.

To prove this, recall that the change of dependent variable, $f=e^{-\gamma x} k=U k$, defined a unitary transformation $U$ from $L^{2}\left(R^{+}, d x\right)$ to $L^{2}\left(R^{+}, e^{2 \gamma x} d x\right)$ under which

$$
\mathcal{D}_{1} k:=\left(U^{-1} \mathcal{D} U\right) k=-\frac{d^{2} k}{d x^{2}}+\left(\gamma^{2}+c\right) k,
$$

so that $\mathcal{D}$ is unitarily equivalent to $\mathcal{D}_{1}$ acting on ordinary $L^{2}\left(R^{+}, d x\right)$. From proposition 5.2, $\mathcal{D}_{1}$ has the same essential spectrum as

$$
\mathcal{D}_{2} k=-\frac{d^{2} k}{d x^{2}}+\gamma^{2} k
$$

and, by Fourier transformation, $\mathcal{D}_{2}$ is unitarily equivalent to the multiplication operator $\mathcal{M}$ in the lemma. The conclusion about the spectrum is immediate.

We are now ready to prove the main result of this section, Proposition 5.1, concerning the spectrum of the Laplacian (on 0-forms and 1-forms) and of the Floer operator (at the zero connection). Recall that we have already shown, in Section 4, the absence of eigenvalues, so that it suffices to obtain the results for the essential spectrum. 
We use $d_{s}$ and $d_{s}^{*}$ to denote, respectively, exterior differentiation and its $L^{2}$ adjoint on $S^{2}$. Let $\Delta_{s}$ denote the Laplacian on $S^{2}$. For notational simplicity, we write $g=\sinh r$ and $w=\cosh ^{2} \beta r$. Sometimes, for clarity, we use the subscriptp 0 or 1 on the Laplacian to distinguish the domain as functions or 1-forms.

On $\mathbb{H}^{3}$, the formulas for the weighted Laplacian are: for $\varphi \in \wedge^{0}$,

$$
\Delta_{0} \varphi=g^{-2} \Delta_{s} \varphi-g^{-2} w^{-1} \frac{\partial}{\partial r}\left(g^{2} w \frac{\partial \varphi}{\partial r}\right)
$$

and for $a=a_{1}+a_{2} d r \in \wedge^{1}$,

$$
\begin{aligned}
\Delta_{1} a= & g^{-2} \Delta_{s} a-w^{-1} \frac{\partial}{\partial r}\left(w \frac{\partial a_{1}}{\partial r}\right)-\frac{\partial}{\partial r}\left(g^{-2} w^{-1} \frac{\partial}{\partial r}\left(g^{2} w a_{2}\right)\right) \wedge d r \\
& -2 g^{-1} \frac{\partial g}{\partial r}\left(d_{s} a_{2}+g^{-2} d_{s}^{*} a_{1} \wedge d r\right)
\end{aligned}
$$

To prove the proposition, we separate variables and expand any $p$-form in eigenfunctions on $S^{2}$. Then, every $\varphi \in \wedge^{0}$ is a sum of terms of the form $h_{0} \tau_{0}$ (with $\tau_{0}$ an eigenfunction on $S^{2}$ ). Using a Hodge decomposition, one sees that every 1 -form on $\mathbb{H}^{3}$ is a sum of three terms (corresponding to the eigenvalue $\lambda$ of $\Delta_{s}$ ) of the form:

$$
h_{1}(r) \tau_{1}+h_{2}(r) \tau_{2} d r+\left(h_{3}(r) d_{s} \tau_{3}+h_{4}(r) \tau_{3} d r\right)
$$

where $\tau_{1}$ is a co-closed eigen 1 -form, and $\tau_{2}$ and $\tau_{3}$ are eigenfunctions on $S^{2}$ (with $\tau_{2}=$ constant corresponding to $\lambda=0$ and $\tau_{3}$ occuring only if $\lambda \neq 0$ ). This decomposition into three types is orthogonal and is preserved both by the Laplacian and the Floer operator.

Note that the forms under consideration will be in $L_{\beta}^{2}\left(\mathbb{H}^{3}\right)$ if and only if

$$
\left\|h_{i}\right\|_{2, \gamma_{i}}^{2}=\int_{0}^{\infty} h_{i}^{2}(r) \gamma_{i}(r) d r<\infty \quad i=0,1,2,3,4
$$

where $\gamma_{0}=\gamma_{2}=\gamma_{4}=g^{2} w$ and $\gamma_{1}=\gamma_{3}=w$.

In this context, the Laplacian defines ordinary differential operators on the spaces in (5.6) as follows:

$$
\begin{aligned}
& \left(\mathcal{D}_{0} h_{0}\right) \tau_{0}=\Delta_{0}\left(h_{0} \tau_{0}\right)=\left(-g^{-2} w^{-1} \frac{d}{d r}\left(g^{2} w \frac{d h_{0}}{d r}\right)+\lambda g^{-2} h_{0}\right) \tau_{0} \\
& \left(\mathcal{D}_{1} h_{1}\right) \tau_{1}=\Delta_{1}\left(h_{1} \tau_{1}\right)=\left(-w^{-1} \frac{d}{d r}\left(w \frac{d h_{1}}{d r}\right)+\lambda g^{-2} h_{1}\right) \tau_{1} \\
& \left(\mathcal{D}_{2} h_{2}\right) \tau_{2} d r=\Delta_{1}\left(h_{2} \tau_{2} d r\right)=\left(-\frac{d}{d r}\left(g^{-2} w^{-1} \frac{d}{d r}\left(g^{2} w h_{2}\right)\right)+\lambda g^{-2} h_{2}\right) \tau_{2} d r \\
& \mathcal{D}_{3}\left(h_{3}, h_{4}\right)=\left(\mathcal{D}_{1} h_{3}+2 g^{-1} \frac{d g}{d r} h_{4}, \mathcal{D}_{2} h_{4}+2 g^{-3} \frac{d g}{d r} h_{3}\right) .
\end{aligned}
$$

The explanation for (iv) is that 


$$
\Delta_{1}\left(h_{3} d_{s} \tau_{3}+h_{4} \tau_{3} d r\right)=\left(\mathcal{D}_{1} h_{3}+2 g^{-1} \frac{d g}{d r} h_{4}\right) d_{s} \tau_{3}+\left(\mathcal{D}_{2} h_{4}+2 g^{-3} \frac{d g}{d r} h_{3}\right) \tau_{3} d r
$$

Note that $\mathcal{D}_{0}$ and $\mathcal{D}_{2}$ are operators on $L^{2}\left(R^{+}, g^{2} w d r\right), \mathcal{D}_{1}$ is an operator on $L^{2}\left(R^{+}, w d r\right)$ and $\mathcal{D}_{3}$ acts on $L^{2}\left(R^{+}, w d r\right) \times L^{2}\left(R^{+}, g^{2} w d r\right)$.

It follows immediately that $\mathcal{D}_{1}$ is of the form (5.3) with $\gamma=\beta$. Hence, $\sigma\left(\mathcal{D}_{1}\right)=\left[\beta^{2}, \infty\right)$.

Using Proposition 5.2, we may replace $g^{2}$ by $e^{2 r}$ and $w$ by $e^{2 \beta r}$ in $\mathcal{D}_{0}$ and $\mathcal{D}_{2}$ without changing the spectra. This gives us an operator $\tilde{\mathcal{D}}$ acting on $h \in L^{2}\left(R^{+}, e^{2(1+\beta) r} d r\right)$ given by:

$$
\tilde{\mathcal{D}} h=-\frac{d}{d r}\left(e^{-2(1+\beta) r} \frac{d}{d r}\left(e^{2(1+\beta) r} h\right)\right)+\lambda e^{-2 r} h,
$$

which is of the form (5.3) with $\gamma=1+\beta$. Hence, $\sigma\left(\mathcal{D}_{0}\right)=\sigma\left(\mathcal{D}_{2}\right)=\left[(1+\beta)^{2}, \infty\right)$.

Finally, in the third case, again using Proposition 3.14, it suffices to consider the spectrum of

$$
\mathcal{D}_{3}^{\prime}\left(h_{3}, h_{4}\right)=\left(\mathcal{D}_{1} h_{3}+2 h_{4}, \mathcal{D}_{2} h_{4}+e^{-2 r} h_{3}\right)
$$

acting on pairs $\left(h_{3}, h_{4}\right) \in L^{2}\left(R^{+}, e^{2 \beta r} d r\right) \times L^{2}\left(R^{+}, e^{2(1+\beta) r} d r\right)$. Making the unitary change of variable $\left(h_{3}, h_{4}\right)=\left(e^{-\beta r} k_{3}, e^{-(1+\beta) r} k_{4}\right)$ gives the operator $\mathcal{D}_{3}^{\prime \prime}=U^{-1} \mathcal{D}_{3}^{\prime} U$ where

$$
\mathcal{D}_{3}^{\prime \prime}\left(k_{3}, k_{4}\right)=\left(\frac{-d^{2} k_{3}}{d r^{2}}+\beta^{2} k_{3}+2 e^{-r} k_{4}, \frac{-d^{2} k_{4}}{d r^{2}}+(1+\beta)^{2} k_{4}+2 e^{-r} k_{3}\right)
$$

on pairs $\left(k_{3}, k_{4}\right) \in L^{2}\left(R^{+}, d r\right) \times L^{2}\left(R^{+}, d r\right)$. Another application of Proposition 5.2 shows that the spectrum of $\mathcal{D}_{3}$ is

$$
\sigma\left(\mathcal{D}_{3}\right)=\left[\beta^{2}, \infty\right) \cup\left[\left(1+\beta^{2}\right), \infty\right)=\left[\beta^{2}, \infty\right) .
$$

Since up to compact perturbation, $\Delta_{p}$ is unitarily equivalent to sums of the above operators, we have demonstrated (a) and (b) of proposition (5.1).

To prove (c), we show that $\delta_{0} \delta_{0}^{\dagger}$ differs from the Laplacian on pairs by a compact perturbation. Recall $(3.16)$ that $\delta_{0} \delta_{0}^{\dagger}(\psi, b)=\left(\Delta_{0} \psi, \Delta_{1} b\right)+\left(0, T_{1} b\right)$

where

$$
T_{1} b=-2 \beta \tanh \beta r\left\{*(d r \wedge * d b)+d^{*}(b \wedge d r)\right\}
$$

Evaluating $T_{1}$ on each of the three types of one forms occurring in (5.5), we find that $T_{1} b=0$ on the subspaces spanned by $\tau_{1}$ and $\tau_{2} d r$. For $b$ of the third kind, $T_{1}$ gives rise to the operator

$$
\mathcal{T}\left(h_{3}, h_{4}\right)=-2 \beta \tanh \beta r\left(h_{4}, \lambda g^{-2} h_{3}\right)
$$


since $T_{1}\left(h_{3} d_{s} \tau_{3}+h_{4} \tau_{3} d r\right)=-2 \beta \tanh \beta r\left(h_{4} d_{s} \tau_{3}+\lambda g^{-2} h_{3} \tau_{3} d r\right)$. As before, $\left(h_{3}, h_{4}\right) \in$ $L^{2}\left(R^{+}, e^{2 \beta r} d r\right) \times L^{2}\left(R^{+}, e^{2(1+\beta) r} d r\right)$. The unitary change of dependent variable $\left(h_{3}, h_{4}\right)=$ $\left(e^{-\beta r} k_{3}, e^{-(1+\beta) r} k_{4}\right)$ gives a unitary equivalence of $\mathcal{T}$ with

$$
\hat{\mathcal{T}}\left(k_{3}, k_{4}\right)=\left(U^{-1} \mathcal{T} U\right)\left(k_{3}, k_{4}\right)=-2 \beta \tanh \beta r\left(e^{-r} k_{4}, \lambda e^{r} g^{-2} k_{3}\right)
$$

acting on $L^{2}\left(R^{+}, d r\right) \times L^{2}\left(R^{+}, d r\right)$.

It follows that $T_{1}$ contributes a compact perturbation to $\Delta_{1}$, and hence, by Prop 5.2 does not change the spectrum from which (c) of proposition 5.1 follows.

\section{The Existence Theorem}

We recall the

Raleigh Quotient Theorem. (cf [Da] Theorem 4.3.1, p. 78) If $L$ is a self-adjoint operator on a Hilbert space $\mathcal{H}$, then $(L f, f)_{\mathcal{H}} \geq c\|f\|_{\mathcal{H}}^{2}$ for all $f$ in the domain of $L$, if and only if the spectrum $\sigma(L) \subset[c, \infty)$.

Then, letting $\Delta=\left(\Delta_{0}, \Delta_{1}\right)$ denote the Laplacian on configuration pairs, the information in Proposition 5.1 may be translated (using the Raleigh-Quotient Theorem) into the inequalities:

$$
\begin{array}{ll}
\left(a^{\prime}, b^{\prime}\right) & \beta^{2}\|\eta\|_{2, \beta}^{2} \leq(\Delta \eta, \eta)_{\beta} \\
\left(c^{\prime}\right) & \beta^{2}\|\eta\|_{2}^{2, \beta} \leq\left(\delta_{0} \delta_{0}^{\dagger} \eta, \eta\right)_{\beta}=\left(\delta_{0}^{\dagger} \eta, \delta_{0}^{\dagger} \eta\right)_{\beta}
\end{array}
$$

Next, we show that the scalar operators are invertible on $\mathcal{H}_{0}$. (Recall that $\mathcal{H}_{0}=L_{\beta}^{2,2}$.)

Proposition 6.2. There is a constant $\kappa>0$ such that

$$
\kappa\|\eta\|_{\mathcal{H}_{0}} \leq\left\|\delta_{0} \delta_{0}^{\dagger} \eta\right\|_{2, \beta}
$$

and $\delta_{0} \delta_{0}^{\dagger}$ is invertible.

Proof. First, we note that since $\delta_{0} \delta_{0}^{\dagger}$ is a self-adjoint operator defined on $\mathcal{H}_{0}$, the inequality implies that the co-kernel is zero and hence, $\delta_{0} \delta_{0}^{\dagger}$ is not only injective but surjective with closed range, and hence invertible.

To prove the inequality, we recall the Bochner-Lichnerowicz-Weitzenbock formula at a point, for the standard Laplacian $\Delta_{p}^{0}=d d^{*}+d^{*} d([\mathrm{FU}])$ :

$$
-\nabla^{2} \omega=\Delta_{p}^{0} \omega-\operatorname{Ric}_{p}(\omega, \cdot), \quad \omega \in \wedge^{p}, p=0,1
$$

and $\mathrm{Ric}_{0}=0$. 
Letting $\nabla^{\dagger} \omega=\cosh ^{-2} \beta r \nabla\left(\left(\cosh ^{2} \beta r\right) \omega\right)$, and, noting that

$$
\Delta_{p} \omega=\left(d d^{\dagger}+d^{\dagger} d\right) \omega=\Delta_{p}^{0} \omega+2 \beta \tanh \beta r(*(d r \wedge * d \omega))+2 \beta d(*(\tanh \beta r d r \wedge * \omega))
$$

we obtain

$$
\begin{aligned}
-\nabla^{\dagger} \nabla \omega= & \Delta_{p} \omega-\operatorname{Ric}_{p}(\omega, \cdot)-2 \beta \tanh \beta r(*(d r \wedge * d \omega)) \\
& -2 \beta d(*(\tanh \beta r d r \wedge * \omega))
\end{aligned}
$$

An integration by parts and use of (6.1) gives

$$
\begin{aligned}
\|\nabla \omega\|_{2, \beta}^{2} & \leq\left(\Delta_{p} \omega, \omega\right)+c\|\nabla \omega\|_{2, \beta}\|\omega\|_{3, \beta}+c^{\prime}\|\omega\|_{2, \beta}^{2} \\
& \leq(1+C(\epsilon))\left(\Delta_{p} \omega, \omega\right)+\epsilon\|\nabla \omega\|_{2, \beta}^{2}
\end{aligned}
$$

from which it follows that

$$
\|\omega\|_{L_{\beta}^{1,2}} \leq C\left\|\Delta_{p} \omega\right\|_{2, \beta}
$$

Using the Weitzenbock formula once again,

$$
\left\|\nabla^{2} \omega\right\|_{2, \beta}^{2} \leq\left\|\Delta_{p} \omega\right\|_{2, \beta}^{2}+C^{\prime}\|\omega\|_{L_{\beta}^{1,2}}^{2} \leq C^{\prime \prime}\left\|\Delta_{p} \omega\right\|_{2, \beta}^{2}
$$

which gives

$$
\|\omega\|_{L_{\beta}^{2,2}} \leq C\left\|\Delta_{p} \omega\right\|_{2, \beta}, \quad \omega \in \wedge^{p} \quad p=0,1
$$

Next, recall again (3.16) that on pairs,

$$
\delta_{0} \delta_{0}^{\dagger} \eta=\Delta \eta+T \eta \text { where } T=\left(0, T_{1}\right)
$$

and $T_{1}$ is defined by (3.12). Using (6.6), (6.1c'), Proposition 3.18 and Sobolev's inequality

$$
\begin{aligned}
\|\eta\|_{\mathcal{H}_{0}} & \leq C\|\Delta \eta\|_{2, \beta} \leq C\left(\left\|\delta_{0} \delta_{0}^{\dagger} \eta\right\|_{2, \beta}+\left\|T_{1} b\right\|_{2, \beta}\right) \\
& \leq C\left\|\delta_{0} \delta_{0}^{\dagger} \eta\right\|_{2, \beta}+C^{\prime}\|b\|_{L_{\beta}^{1,2}} \\
& \leq C\left\|\delta_{0} \delta_{0}^{\dagger} \eta\right\|_{2, \beta}+C(\epsilon)\|\eta\|_{2, \beta}+\epsilon\|\eta\|_{\mathcal{H}_{0}} \\
& \leq C^{\prime}\left\|\delta_{0} \delta_{0}^{\dagger} \eta\right\|_{2, \beta}+\epsilon\|\eta\|_{\mathcal{H}_{0}}
\end{aligned}
$$

Absorbing the term $\epsilon\|\eta\|_{\mathcal{H}_{0}}$, proves the inequality of Proposition 6.2.

Finally, letting $\mathcal{L}=\delta \delta^{\dagger}$, (recall the definitions of $\delta$ and $\delta^{\dagger}$ from (3.4) and (3.7)) we are now ready to prove the main estimate of this paper.

Theorem 6.7. There is a constant $\alpha_{1}>0$ such that

$$
\alpha_{1}\|\eta\|_{\mathcal{H}_{A_{0}}} \leq\|\mathcal{L} \eta\|_{2, \beta} \text { and } \mathcal{L} \text { is invertible. }
$$


We notice that, as before, $\mathcal{L}$ is self-adjoint on its domain $\mathcal{H}_{A_{0}}$ and hence, the inequality shows invertibility.

To prove the theorem, we compare the various operators evaluated at $A_{0}$ with their scalar analogues to show that the lower bounds on spectra do not decrease.

We note first that from (3.13) we have that $\Delta_{A_{0}}=\Delta+S$ with $S$ rapidly decaying at infinity. Propositions (3.18) and (5.2) then imply

$$
\sigma\left(\Delta_{A_{0}}\right)=\sigma_{e s s}\left(\Delta_{A_{0}}\right) \subseteq\left[\beta^{2}, \infty\right)
$$

Similarly, we recall (3.17) $\delta_{A_{0}} \delta_{A_{0}}^{\dagger}=\delta_{0} \delta_{0}^{\dagger}+R+S$.

Again Propositions (3.18) and (5.2) imply

$$
\sigma\left(\delta_{A_{0}} \delta_{A_{0}}^{\dagger}\right)=\sigma_{e s s}\left(\delta_{A_{0}} \delta_{A_{0}}^{\dagger}\right) \subseteq\left[\beta^{2}, \infty\right)
$$

Finally, recalling (3.8), $\delta \delta^{\dagger}=\delta_{A_{0}} \delta_{A_{0}}-(a d \Phi)^{2}+E$, Propositions (3.18) and (5.2), together with the observation that $-\left(a d \Phi_{0}\right)^{2}$ is a non-negative operator, result in

$$
\sigma\left(\delta \delta^{\dagger}\right)=\sigma_{e s s}\left(\delta \delta^{\dagger}\right) \subseteq\left[\beta^{2}, \infty\right)
$$

Using the Raleigh-Quotient Theorem,

$$
\left\{\begin{array}{l}
\beta^{2}\|\eta\|_{2, \beta}^{2} \leq\left(\Delta_{A_{0}} \eta, \eta\right)_{\beta} \\
\kappa\|\eta\|_{2, \beta}^{2} \leq\left(\delta \delta^{\dagger} \eta, \eta\right)_{\beta} \text { for some } \kappa \geq \beta^{2} .
\end{array}\right.
$$

To prove the inequality in the theorem, we use the Weitzenbock formula at $A_{0}$, with $\beta=0$ to obtain

$$
d_{A_{0}}^{*} d_{A_{0}}+d_{A_{0}} d_{A_{0}}^{*}=-\nabla_{A_{0}}^{2}+\{R i c, \cdot\}+\left\{F_{A_{0}}, \cdot\right\} .
$$

For the weighted operators, with $\beta>0$, we find

$$
\begin{aligned}
& \Delta_{A_{0}}=d_{A_{0}}^{\dagger} d_{A_{0}}+d_{A_{0}} d_{A_{0}}^{\dagger}=-\nabla_{A_{0}}^{\dagger} \nabla_{A_{0}}+Q_{1} \\
& \delta \delta^{\dagger}=-\nabla_{A_{0}}^{\dagger} \nabla_{A_{0}}+Q_{2}
\end{aligned}
$$

where $\left|Q_{i} \eta\right| \leq c(|\eta|+|\nabla \eta|), i=1,2$

As before, 


$$
\begin{aligned}
\left\|\nabla_{A_{0}} \eta\right\|_{2, \beta}^{2} & \leq\left(\delta \delta^{\dagger} \eta, \eta\right)_{\beta}+\left(Q_{2} \eta, \eta\right)_{\beta} \\
& \leq\left(\delta \delta^{\dagger} \eta, \eta\right)_{\beta}+\epsilon\left\|\nabla_{A_{0}} \eta\right\|_{2, \beta}^{2}+C(\epsilon)\|\eta\|_{2, \beta}^{2}
\end{aligned}
$$

and therefore, from (6.11),

$$
\|\eta\|_{2, \beta}+\left\|\nabla_{A_{0}} \eta\right\|_{2, \beta} \leq C^{\prime}\left\|\delta \delta^{\dagger} \eta\right\|_{2, \beta}
$$

Using (6.6), (6.1c), Proposition 3.18 and Sobolev's inequality prove the theorem.

Note that because of the explicit knowledge about the Chakrabarti monopole, the decay of the approximate monopole is known for $\mathbb{H}^{3}$ and also that the basic estimate, Theorem (6.7) holds without any assumption that $L c_{0}=d_{A_{0}} \Phi_{0}-* F_{A_{0}}$ is small. However, this condition will be needed later in applying the Implicit Function Theorem. The restriction $\beta<m$, required to make $L c_{0}$ small, will also be used later.

Corollary 6.16. If $\|\nu\|_{6, \beta}$ is sufficiently small, then

$$
\mathcal{L}_{\nu}=\mathcal{L}+\nu \# \delta^{\dagger}
$$

is invertible.

Proof. We use a weighted version of Sobolev's inequality which says that, for $2<p \leq 6$,

$$
\|\eta\|_{p, \beta} \leq C\left(\left\|\nabla_{A_{0}} \eta\right\|_{2, \beta}+\|\eta\|_{2, \beta}\right)
$$

This inequality implies that

$$
\|\eta\|_{p, \beta} \leq C\|\eta\|_{\mathcal{H}_{A_{0}}} \text { and }\left\|\zeta^{\dagger} \eta\right\|_{p, \beta} \leq C^{\prime}\|\eta\|_{\mathcal{H}_{A_{0}}}
$$

Using the Holder's inequality, with $\alpha_{2}=\max \left(C, C^{\prime}\right)$,

$$
\begin{aligned}
& \left\|v \# \delta^{\dagger} \eta\right\|_{2, \beta} \leq\|v\|_{6, \beta}\left\|\delta^{\dagger} \eta\right\|_{3, \beta} \leq \alpha_{2}\|v\|_{6, \beta}\|\eta\|_{\mathcal{H}_{A_{0}}} \leq \alpha_{2}^{2}\|v\|_{\mathcal{H}_{A_{0}}}\|\eta\|_{\mathcal{H}_{A_{0}}} \\
& \left\|\delta^{\dagger} \tau \# \delta^{\dagger} \eta\right\|_{2, \beta} \leq \alpha_{2}^{2}\|\tau\|_{\mathcal{H}_{A_{0}}}\|\eta\|_{\mathcal{H}_{A_{0}}}
\end{aligned}
$$

Using Theorem 6.7,

$$
\alpha_{1}\|\eta\|_{\mathcal{H}_{A_{0}}} \leq\left\|\mathcal{L}_{\nu} \eta\right\|_{2, \beta}+\left\|\nu \# \delta^{\dagger} \eta\right\|_{2, \beta},
$$

which combined with (6.18) gives, for $\|\nu\|_{6, \beta}$ sufficiently small,

$$
\alpha^{\prime}\|\eta\|_{\mathcal{H}_{A_{0}}} \leq\left\|\mathcal{L}_{\nu} \eta\right\|_{2, \beta}
$$

Invertibility of $\mathcal{L}_{\nu}$, for $\|\nu\|_{6, \beta}$ small, follows from the fact that $\mathcal{L}_{0}$ is invertible and $\| \mathcal{L}_{0}-$ $\mathcal{L}_{v}\left\|_{2, \beta} \leq C\right\| \nu \|_{6, \beta}$.

Recall that we have seen in section 3, that to solve the Bogomolny monopole equation

$$
L c=L(\Phi, A)=d_{A} \Phi-* F_{A}=0
$$


it suffices to find a solution $\eta=(\psi, b)$ of

$$
\mathscr{L} \eta=\delta \delta^{\dagger} \eta+\delta^{\dagger} \eta \# \delta^{\dagger} \eta=G_{0}
$$

for $G_{0}$ and $\delta^{\dagger} \eta \# \delta^{\dagger} \eta$ sufficiently small.

Theorem. The Bogomolny monopole equation (1.1) has a solution

$c=(\Phi, A)$ with given magnetic charge $k \in Z$ and (arbitrary) mass $m \in \mathbb{R}^{+}$.

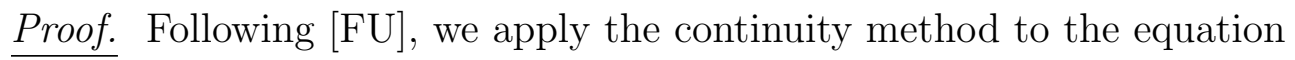

$$
\mathscr{L} \eta_{t}=\delta \delta^{\dagger} \eta_{t}+\delta^{\dagger} \eta_{t} \# \delta^{\dagger} \eta_{t}=t G_{0}, \quad 0 \leq t \leq 1 .
$$

To that end, let $\lambda<\alpha_{1} / 4 \alpha_{2}^{2}$ where $\alpha_{1}$ and $\alpha_{2}$ are the constants occurring in the inequalities (6.7) and (6.18). Also, assume that $\left\|G_{0}\right\|_{2, \beta} \leq \alpha_{1} \lambda / 4$.

Let

$$
\Omega=\left\{\eta \in \mathcal{H}_{A_{0}}:\|\eta\|_{\mathcal{H}_{A_{0}}} \leq \lambda\right\}
$$

and

$$
J=\{t \in[0,1] \text { equation }(*) \text { has a solution in } \Omega\}
$$

We show that $J$ is non-empty, open and closed. Clearly, $t=0$ belongs to $J$ since $\eta \equiv 0$ is the unique solution.

To show that $J$ is open, let $t_{0} \in J$, with $\eta_{0}$ the corresponding solution of (*) belonging to $\Omega$. The linearized operator at $\eta_{0}$ is $\mathcal{L}_{\nu}$ with $\nu=2 \delta^{\dagger} \eta_{0}$. From (6.16) and (6.17) at $2 \delta^{\dagger} \eta_{0}$ and the choice of $\lambda$ above, one has $\left\|\nu \# \delta^{\dagger} \eta\right\|_{2, \beta} \leq \frac{\alpha_{1}}{2}\|\eta\|_{\mathcal{H}_{A_{0}}}$. It follows from (6.18) that (6.20) holds with $\alpha^{\prime}=\frac{\alpha_{1}}{2}$ and $\mathcal{L}_{\nu}$ is invertible.

From the Implicit Function Theorem, we conclude that $(*)$ has a solution $\eta_{t}$ for $t$ sufficiently close to $t_{0}$, and $\left\|\eta_{t}-\eta_{0}\right\|_{\mathcal{H}_{A_{0}}}<\epsilon$, for $\epsilon$ sufficiently small. Estimating again (as in 6.18), and using the fact that $\eta_{0} \in \Omega$, we find, from (6.7)

$$
\begin{aligned}
\alpha_{1}\left\|\eta_{0}\right\|_{\mathcal{H}_{A_{0}}} \leq\left\|t_{0} G_{0}\right\|_{2, \beta}+\left\|\delta^{\dagger} \eta_{0} \# \delta^{\dagger} \eta_{0}\right\|_{2, \beta} \\
\leq\left\|t_{0} G_{0}\right\|_{2, \beta}+\frac{\alpha_{1}}{4}\left\|\eta_{0}\right\|_{\mathcal{H}_{A_{0}}}
\end{aligned}
$$

Using the bound on $G_{0}$ from Proposition 2.7, we find

$$
\left\|\eta_{0}\right\|_{\mathcal{H}_{A_{0}}} \leq \frac{1}{3} \lambda
$$

so that for $\eta_{t}$ sufficiently close to $\eta_{0}$, which will be the case if $t$ is close to $t_{0}$, we see that

$$
\left\|\eta_{t}\right\|_{\mathcal{H}_{A_{0}}} \leq \lambda
$$

Therefore, $J$ is open. 
To prove that $J$ is closed, let $t_{n} \in J$ converge to $t_{0}$. Then, for each $n$, let $\eta_{n}$ be the solution of $(*)$ corresponding to $t_{n}$. Since $\left\|\eta_{n}\right\|_{\mathcal{H}_{\mathcal{A}}} \leq \lambda$, a subsequence converges weakly in $\mathcal{H}_{\mathcal{A}_{0}}$ to $\eta_{0}$ and by lower semi-continuity with respect to weak convergence, $\left\|\eta_{0}\right\|_{\mathcal{H}_{\mathcal{A}_{0}}} \leq \lambda$. We claim that $\mathscr{L} \eta_{0}=t_{0} G_{0}$. It suffices to show this on any compact subdomain. The linear term $\delta \delta^{\dagger} \eta_{n}$ converges weakly to $\delta \delta^{\dagger} \eta_{0}$. By Sobolev embedding $\delta^{\dagger} \eta_{n}$ converges strongly to $\delta^{\dagger} \eta_{0}$ in $L_{\beta}^{p}$ for $p<6$ and therefore, $\delta^{\dagger} \eta_{n} \# \delta^{\dagger} \eta_{n}$ converges strongly to $\delta^{\dagger} \eta_{0} \# \delta^{\dagger} \eta_{0}$ in $L_{\beta}^{2}$, since $\left\|\delta^{\dagger} \eta_{0} \# \delta^{\dagger} \eta_{0}\right\|_{2, \beta} \leq\left\|\delta^{\dagger} \eta_{0}\right\|_{4, \beta}^{2}$ and Lebesgue dominated convergence is applicable. It follows that $\mathscr{L} \eta_{n}$ converges weakly to $\mathscr{L} \eta_{0}$ which is a solution of the equation, as desired. This shows that $\mathrm{J}$ is closed, and completes the proof of our main theorem. 
[A] Atiyah, M.F., Magnetic monopoles in hyperbolic spaces: Vector bundles on algebraic varieties, Tata Institute of Fundamental Research, Bombay (1984), 1-33.

[Br] Braam, P.J., Magnetic monopoles on three-manifolds, J. Diff. Geom. 30 (1989), 425-464.

[Bo] Bogomolny, E.B., The stability of classical solutions, Sov. J. Nucl. Phys. 24 (1976), 449.

[C] Chakrabarti, A., Spherically and axially symmetric SU(n) instanton chains with monopole limits, Nucl. Phys. B 248 (1984), 209-252.

[D] Donaldson, S.K., Anti-self-dual Yang Mills connections over complex algebraic surfaces and stable vector bundles, Proc. Lond. Math. Soc. 30 (1985), 1-26.

[Da] Davies, E.B., Spectral Theory and Differential Operators, Cambridge Univ. Press (1995).

[Di] Dirac, P.A.M., Proc Roy Soc, A 133, 60 (1931)

[Do] Donnelly, H., Differential Form Spectrum of Hyperbolic space, Manuscripta Math. 33 (1981), 365-385.

[Du] Durenard, E., Mayer Vietoris Result for Monopoles, Harvard Ph. D. thesis (1995).

[E] Ernst, K.D., The ends of the Monopole Moduli space over $\mathbb{R}^{3} \#$ (Homology sphere): I and II, The Floer Memorial Volume, Birkhauser (1995), 355-434.

[F1] Floer, A., Monopoles on asymptotically Euclidean 3-manifolds., Bull. Amer. Math. Soc. 16 (1987), 125-127.

[F2] Floer, A., Monopoles on asymptotically flat manifolds, preprint (1987), The Floer Memorial Volume, Birkhauser (1995), 3-42.

[FHP1] Forgacs, P., Horvath, Z., Palla, L., An exact fractionally charged self-dual solution, Phys. Rev. Lett. 46 (1981), 392.

[FHP2] Forgacs, P., Horvath, Z., Palla, L., One can have non-integer topological charge, Z. Phys. C 12 (1982), 359-360.

[FU] Freed, D.S., Uhlenbeck, K., Instantons and Four Manifolds, Mathematical Sciences Research Institute Publications 1 (1984), Springer-Verlag.

[G] Giaquinta, M., Multiple Integrals in the Calculus of Variations, Annals of Mathematics Studies, Study 105 (1983).

[H] Harland, D., Hyperbolic calorons, monopoles, and instantons, Comm. Math. Phys. 280 (2008) 727-735.

[JT] Jaffe, A., Taubes, C., Vortices and Monopoles, Birkhauser Progress in Physics 2 (1980). 
[L] Landweber, G.D., Singular instantons with so(3) symmetry, arXiv:math.dg/0503611 (2005).

[M] Mazzeo, R., Unique continuation at infinity and embedded eigenvalues for asymptotically hyperbolic manifolds, Amer. J. Math 113 (1991) 25-45.

[MS] Manton, N., Sutcliffe, P., Topological Solitions, Cambridge (2004).

[PS] Prasad, M. K., Sommerfield, C. M., Exact classical solutions for the 't Hooft monopole and the Julia Zee dyon, Phys. Rev. Lett. 35 (1975) 760-762.

[R] Rade, J., On singular Yang-Mills fields: gauge fixing and growth estimates, preprint (1993).

[RS] Reed, M., Simon, B., Analysis of Operators IV, Academic Press (1978).

[SS1] Sibner, L.M., Sibner, R.J., Singular Sobolev connections with holonomy, Bull. Amer. Math. Soc. 19 (1988), 471-473.

[SS2] Sibner, L.M., Sibner, R.J., Classification of singular Sobolev connections by their holonomy, Comm. Math. Phys. 144 (1992), 337-350.

[SSU] Sibner, L.M., Sibner, R.J., Uhlenbeck, K., Solutions to Yang-Mills Equations that are not self-dual, Proc. Nat. Acad. Sci., USA 86 (1989), 8610-8613.

[T] Taubes, C.H., Self-dual Yang-Mills connections on non-self-dual 4-manifolds, J. Diff. Geom. 17 (1982), 139-170. 